\title{
Nosocomial infections associated to COVID-19 in the intensive care unit: clinical characteristics and outcome
}

\author{
Tommaso Bardi ${ }^{1}$ (D) Vicente Pintado ${ }^{2}$ - Maria Gomez-Rojo ${ }^{1} \cdot$ Rosa Escudero-Sanchez $^{2} \cdot$ Amal Azzam Lopez $^{1}$. \\ Yolanda Diez-Remesal ${ }^{1} \cdot$ Nilda Martinez Castro ${ }^{1} \cdot$ Patricia Ruiz-Garbajosa $^{3} \cdot$ David Pestaña $^{1}$
}

Received: 22 September 2020 / Accepted: 21 December 2020 / Published online: 3 January 2021

(C) The Author(s), under exclusive licence to Springer-Verlag GmbH, DE part of Springer Nature 2021

\begin{abstract}
Bacterial and fungal co-infection has been reported in patients with COVID-19, but there is limited experience on these infections in critically ill patients. The objective of this study was to assess the characteristics and ouctome of ICU-acquired infections in COVID-19 patients. We conducted a retrospective single-centre, case-control study including 140 patients with severe COVID-19 admitted to the ICU between March and May 2020. We evaluated the epidemiological, clinical, and microbiological features, and outcome of ICU-acquired infections. Fifty-seven patients $(40.7 \%)$ developed a bacterial or fungal nosocomial infection during ICU stay. Infection occurred after a median of 9 days (IQR 5-11) of admission and was significantly associated with the APACHE II score $(p=0.02)$. There were 91 episodes of infection: primary $(31 \%)$ and catheter-related $(25 \%)$ bloodstream infections were the most frequent, followed by pneumonia (23\%), tracheobronchitis $(10 \%)$, and urinary tract infection $(8 \%)$ that were produced by a wide spectrum of Gram-positive (55\%) and Gramnegative bacteria (30\%) as well as fungi (15\%). In $60 \%$ of cases, infection was associated with septic shock and a significant increase in SOFA score. Overall ICU mortality was 36\% (51/140). Infection was significantly associated with death (OR 2.7, 95\% CI 1.2-5.9, $p=0.015)$ and a longer ICU stay $(p<0.001)$. Bacterial and fungal nosocomial infection is a common complication of ICU admission in patients with COVID-19. It usually presents as a severe form of infection, and it is associated with a high mortality and longer course of ICU stay.
\end{abstract}

Keywords COVID-19 $\cdot$ SARS-CoV-2 $\cdot$ Nosocomial infection $\cdot$ Intensive care $\cdot$ Outcome

Nosocomial infection during ICU stay is a common occurrence in severe COVID-19 patients, and it is associated with increased mortality and a longer course of hospitalization.

Tommaso Bardi tommaso.bardi@gmail.com

1 Department of Anaesthesia and Intensive Care, Hospital Universitario Ramón y Cajal, Instituto Ramón y Cajal de Investigación Sanitaria (IRYCIS), M-607, km. 9, 100, 28034 Madrid, Spain

2 Department of Infectious Diseases, Hospital Universitario Ramón y Cajal, Instituto Ramón y Cajal de Investigación Sanitaria (IRYCIS), Madrid, Spain

3 Servicio de Microbiología, Hospital Universitario Ramón y Cajal, Instituto Ramón y Cajal de Investigación Sanitaria (IRYCIS), Madrid, Spain

\section{Introduction}

The pandemic of SARS-CoV-2 infection at the beginning of 2020 has heavily hit most countries in the world, and one of the major challenges imposed by this infection has been the large numbers of patients in need for intensive care [1-3]. Bacterial and fungal superinfection during intensive care unit (ICU) stay has been reported in other outbreaks of severe acute respiratory syndrome (SARS), but there is limited data available regarding COVID-19 patients. Many authors recognize the importance of superinfection but definitive data is still lacking $[4,5]$. Reported incidence varies between 3.6 and $43 \%$ [6], and no study thus far has focused specifically on the subpopulation of ICU patients.

Moreover, the role of the host response to infection by SARS-CoV-2 in COVID-19 disease has been used as a potential target for therapy, and a number of immunomodulatory treatments have been proposed throughout the outbreak. Steroid therapy, with varying doses and regimens, specific 
biologic drugs such as tocilizumab, and a number of other repurposed drugs such as hydroxychloroquine and azithromycin are all examples of attempts at immunomodulation to fight the devastating effects of COVID-19. Evidence on the efficacy and on the possible side effects of these drugs is only starting to emerge now, after a widespread use of these therapies has been done during the peak of the outbreak. This specific aspect of COVID-19 therapy represents a new challenge for most physicians involved in intensive care medicine (ICM). In particular, there is scarce evidence regarding the possible involvement and interaction of SARS-CoV-2 and its specific therapeutic regiments in the development of hospital-acquired infections.

In this study, we investigated the clinical features of bacterial and fungal infections associated with COVID-19 in ICU patients, their microbiological characteristics, their impact on the course of critical illness, and the possible relation with risk factors.

\section{Materials and methods}

\section{Study design and setting}

We conducted a retrospective study of all patients hospitalized in the intensive care units at a tertiary university hospital in the city of Madrid, with a confirmed diagnosis of COVID-19, during the months of March, April, and May 2020. The study was approved by the hospital ethics committee and complies with the principles of the Helsinki Declaration and its later amendments.

\section{Study population}

We included in the study all adult patients with a positive SARS-CoV-2 polymerase chain reaction (PCR) in nasal or respiratory tract samples, who were hospitalized in the ICU between the 1st of March and the 30th of May 2020. We excluded from the analysis patients who were transferred from other centres and already had a confirmed diagnosis of nosocomial infection at the time of transfer.

\section{Data collection and definitions}

We collected the following data from the electronic medical records: demographic characteristics, medical comorbidities, SOFA score, APACHE II score, source of infection, bacterial and fungal species, severity of systemic inflammatory response, and clinical outcome. We only included in the study cases with confirmed infection, defined by the presence of a positive culture of a significant clinical sample, associated with clinical signs of infection and/or worsening organ failure. Conventional microbiological testing (tracheal aspirate, blood and urine cultures) was requested by the treating physician when infection was suspected, and was not protocolized. Cases were reviewed by an Infectious Diseases specialist and an Anaesthesia specialist to determine the presence of true clinical co-infection and its source. All infections were defined according to the Centers for Disease Control and Prevention criteria and the Spanish Society of Infectious Diseases and Clinical Microbiology [7, 8]; the severity of infection and the presence of septic shock followed Sepsis-3 definitions [9]. If a diagnosis of co-infection was made within the first $48 \mathrm{~h}$ since hospital admission, these infections were defined as community-acquired. If diagnosis occurred $\geq 48 \mathrm{~h}$ from admission for COVID-19, these infections were defined as hospital-acquired superinfections. All patients were treated according to the institution COVID-19 protocol and all the revisions published throughout the outbreak following the appearance of new scientific evidence regarding treatment drugs. Moreover, the hospital ICUs apply the care bundles of the national Spanish guidelines with regard to IV lines and tracheal tube handling $[10,11]$. Microorganisms were defined as multidrug resistant (MDR) if they were resistant to $\geq 1$ drug in at least 3 classes of antibiotics, and XDR if resistant to $\geq 1$ drug in all but $\leq 2$ classes of antibiotics [12]. Outcome variables were ICU mortality, and mortality at hospital discharge, which was recorded for all patients.

\section{Statistical analysis}

Data are presented as median and interquartile range (IQR) for continuous variables, and categorical data are represented as numbers (\%). Chi-squared tests were used for categorical variables and $T$ test for continuous variables. Univariate logistic regression analyses were performed using the occurrence of infection in the ICU as the dependent variable and the candidate risk factors as independent variables; variables with a $p<$ 0.1 were included in a multivariate model built with a stepwise removal of the less significant variable. A second logistic regression was performed using death as a response variable with the same stepwise strategy. Statistical significance was defined as a $p$ value $\leq 0.05$. Statistical analysis was performed with STATA (version 16.1).

\section{Results}

We analyzed the records of 140 patients who fulfilled inclusion criteria for the study. Clinical characteristics of the patients are shown in Table 1 which compares patients who developed a nosocomial infection with patients who did not. 
Table 1 Clinical characteristics of the study population and comparison between infected and non-infected patients

\begin{tabular}{|c|c|c|c|c|}
\hline & $\begin{array}{l}\text { Patients } \\
(N=140)\end{array}$ & $\begin{array}{l}\text { Nosocomial infection during ICU } \\
(N=57)\end{array}$ & $\begin{array}{l}\text { No infection during ICU } \\
(N=83)\end{array}$ & $p$ value \\
\hline Age-year (IQR) & $61(57-67)$ & $63(60-68)$ & $61(54-66)$ & 0.03 \\
\hline Sex-male & $108(77 \%)$ & $47(82 \%)$ & $61(73 \%)$ & 0.21 \\
\hline Body mass index (IQR) & $30.4(26-32)$ & $30.7(26-32)$ & $30(26-31)$ & 0.56 \\
\hline APACHE II (IQR) & $14(10-17)$ & $15(12-19)$ & $13(9-16)$ & 0.02 \\
\hline \multicolumn{5}{|l|}{ Comorbidities } \\
\hline Hypertension & $60(42 \%)$ & $26(45 \%)$ & $38(46 \%)$ & 0.9 \\
\hline Chronic ischemic heart disease & $20(14 \%)$ & $11(19 \%)$ & $9(11 \%)$ & 0.16 \\
\hline Chronic kidney disease & $8(6 \%)$ & $5(9 \%)$ & $3(4 \%)$ & 0.2 \\
\hline Chronic obstructive pulmonary disease & $10(7 \%)$ & $7(12 \%)$ & $6(7 \%)$ & 0.31 \\
\hline Diabetes & $28(20 \%)$ & $16(28 \%)$ & $12(14 \%)$ & 0.048 \\
\hline $\mathrm{PaO}_{2} / \mathrm{FIO}_{2}$ ratio on first day of $\mathrm{MV}$ (IQR) & $124(69-156)$ & $115(60-143)$ & $134(71-210)$ & 0.35 \\
\hline Treated with invasive mechanical ventilation & $134(96 \%)$ & $56(98 \%)$ & $78(94 \%)$ & 0.22 \\
\hline $\begin{array}{l}\text { Time from hospital to ICU admission—-days } \\
\text { (IQR) }\end{array}$ & $4(1-6)$ & $4(1-4)$ & $4(1-6)$ & 0.8 \\
\hline ICU length of hospitalization — days (IQR) & $14(8-17)$ & $20(11-24)$ & $11(7-15)$ & $<0.001$ \\
\hline Severe ARDS at ICU admission & $83(59 \%)$ & $39(68 \%)$ & $44(53 \%)$ & 0.068 \\
\hline Ceftriaxone & $120(85 \%)$ & $53(92 \%)$ & $67(80 \%)$ & 0.042 \\
\hline Azithromycin & $118(84 \%)$ & $53(92 \%)$ & $65(76 \%)$ & 0.019 \\
\hline Other antibiotics & $105(75 \%)$ & $47(82 \%)$ & $58(60 \%)$ & 0.091 \\
\hline Steroids & $127(90 \%)$ & $56(98 \%)$ & $71(85 \%)$ & 0.01 \\
\hline Tocilizumab & $96(68 \%)$ & $40(70 \%)$ & $56(67 \%)$ & 0.73 \\
\hline \multicolumn{5}{|l|}{ Mortality and causes of death } \\
\hline ICU mortality & $51(36 \%)$ & $31(54 \%)$ & $20(24 \%)$ & $<0.001$ \\
\hline Refractory respiratory failure & $19(37 \%)$ & $9(29 \%)$ & $10(50 \%)$ & 0.52 \\
\hline Septic shock & $17(33 \%)$ & $17(55 \%)$ & 0 & $<0.001$ \\
\hline Multiorgan failure & $7(14 \%)$ & $1(14 \%)$ & $6(86 \%)$ & 0.14 \\
\hline Cardiac arrest & $6(12 \%)$ & $3(10 \%)$ & $3(15 \%)$ & 0.63 \\
\hline Other causes & $2(4 \%)$ & $1(3 \%)$ & $1(5 \%)$ & 0.78 \\
\hline
\end{tabular}

Data are presented as number and $\%$, unless otherwise indicated. The most commonly used steroid dose regimen was methylprednisolone $1 \mathrm{mg} / \mathrm{kg} / \mathrm{day}$ for a median of 10 days

$I Q R$, interquartile range; $A R D S$, acute respiratory distress syndrome; $I C U$, intensive care unit

\section{Demographic and epidemiological data}

The median age was 61 years (IQR, 57-67) and the majority were male (77\%). Hypertension (42\%) and diabetes (20\%) were the most frequent underlying diseases. Severe pneumonia was the main cause of ICU admission in most patients, which was complicated with severe acute respiratory distress syndrome (ARDS) in 83 (59\%) of them. The vast majority of patients $(N=134,96 \%)$ required invasive mechanical ventilation during the course of their ICU stay. Six patients $(4.3 \%)$ had a previous episode of bacterial co-infection at the time of ICU admission.

During their stay in the ICU, 57 patients (40.7\%) developed at least one confirmed nosocomial infection. We excluded from the study 133 cases of positive cultures (42 blood cultures, 28 urine cultures, 33 tracheal aspirates, and 28 catheter tip cultures) that were considered contaminations.

\section{Source of infection and microbiology}

We recorded a total of 91 episodes of confirmed nosocomial infection occurring in 57 patients during ICU stay ( 15 patients had 2 different episodes of infection, three patients had 3 , three patients had 4, and one patient had 5). The median time from ICU admission to the onset of the first nosocomial infection was 9 days (IQR 5-11).

Data regarding the clinical features and type of infection are shown in supplementary table 1 . We observed 30 episodes of lower respiratory tract infection (LRTI), of which 21 were 
Table 2 Microbiological isolates by type of infection

\begin{tabular}{|c|c|}
\hline Bacterial/fungal co-infections & $N=91$ \\
\hline Primary bloodstream infection & $N=28(31 \%)$ \\
\hline Enterococcus faecium & $12(43 \%)$ \\
\hline Enterococcus faecalis & $6(21 \%)$ \\
\hline Coagulase-negative staphylococci & $3(11 \%)$ \\
\hline Pseudomonas aeruginosa & $2(7 \%)$ \\
\hline Staphylococcus aureus (methicillin-resistant) & $1(3.5 \%)$ \\
\hline Klebsiella oxytoca & $1(3.5 \%)$ \\
\hline Serratia marcescens & $1(3.5 \%)$ \\
\hline Bacteroides spp. & $1(3.5 \%)$ \\
\hline Candida glabrata & $1(3.5 \%)$ \\
\hline Catheter-related bloodstream infection & $N=24(25 \%)$ \\
\hline Coagulase-negative staphylococci & $13(54 \%)$ \\
\hline Enterococcus faecium & $4(17 \%)$ \\
\hline Candida albicans & $4(17 \%)$ \\
\hline Enterococcus faecalis & $2(8 \%)$ \\
\hline Staphylococcus aureus (methicillin-resistant) & $1(4 \%)$ \\
\hline Ventilator-associated pneumonia & $N=21(23 \%)$ \\
\hline Pseudomonas aeruginosa & $8(38 \%)$ \\
\hline Staphylococcus aureus (methicillin-resistant) & $5(24 \%)$ \\
\hline Aspergillus fumigatus & $2(9 \%)$ \\
\hline Stenotrophomonas maltophilia & $2(9 \%)$ \\
\hline Acinetobacter baumannii & $1(5 \%)$ \\
\hline Aspergillus terreus & $1(5 \%)$ \\
\hline Enterobacter cloacae & $1(5 \%)$ \\
\hline Hafnia alvei & $1(5 \%)$ \\
\hline Hospital-acquired pneumonia/tracheobronchitis & $N=9(10 \%)$ \\
\hline Pseudomonas aeruginosa & $3(33 \%)$ \\
\hline Staphylococcus aureus (methicillin-resistant) & $2(21 \%)$ \\
\hline Aspergillus fumigatus & $1(9 \%)$ \\
\hline Haemophilus influenzae & $1(9 \%)$ \\
\hline Staphylococcus aureus (methicillin-susceptible) & $1(9 \%)$ \\
\hline Stenotrophomonas maltophilia & $1(9 \%)$ \\
\hline Urinary tract infection & $N=7(8 \%)$ \\
\hline Enterococcus faecalis & $3(44 \%)$ \\
\hline Enterococcus faecium & $2(28 \%)$ \\
\hline Pseudomonas aeruginosa & $1(14 \%)$ \\
\hline Acinetobacter baumannii & $1(14 \%)$ \\
\hline Soft tissue infection & $N=2(2 \%)$ \\
\hline Klebsiella pneumoniae & $1(50 \%)$ \\
\hline Enterobacter cloacae & $1(50 \%)$ \\
\hline
\end{tabular}

ventilator-associated pneumonia (VAP), 28 episodes of primary bloodstream infections (BSI), 24 episodes of catheter-related bloodstream infections (CRBSI), 7 urinary tract infections
(UTI), and 2 soft tissue infections. The first episode of infection in the ICU was bacteraemia in 35 patients (19 primary BSI and 16 CRBSI), LRTI in 17 patients (11 VAP), and UTI in 6 cases. The median SOFA score at the diagnosis of infection was 6 (IQR 5-8). Fifty-five episodes of infection (60.4\%) occurring in 38 patients were complicated by septic shock.

The microbiology of the infections is described in Table 2. The most frequent bacterium among patients with primary BSI was Enterococcus faecium (43\%), followed by Enterococcus faecalis $(21 \%)$ and coagulase-negative staphylococci (CNS) $(11 \%)$. Gram-positive bacteria also were the most common cause of CRBSI (CNS 54\%, E. faecium 17\%, E. faecalis 8\%), followed by Candida albicans (17\%). Gram-negative microorganisms were the most frequent cause of LRTI, being Pseudomonas aeruginosa the most common isolated bacteria among patients with VAP (38\%) and tracheobronchitis (33\%). Staphylococcus aureus was also frequently isolated in patients with VAP (24\%) and tracheobronchitis (33\%), most of whom (87\%) were resistant to methicillin (7/8). Aspergillus spp. were isolated in 3 cases of LRTI. As in the case of BSI, E. faecium (44\%) and E. faecalis (28\%) were the most common cause of UTI. Enterobacterales and non-fermenting Gram-negative bacilli such as Acinetobacter baumannii and Stenotrophomonas maltophilia were occasionally isolated as a cause of bacteraemia, LRTI, UTI, and soft tissue infections (Table 2). A total of 28 (31\%) episodes of infection, occurring in 22 patients, were sustained by MDR microorganisms and 3 episodes of infection by XDR microorganisms. The most frequent resistant microorganisms were MRSA ( $n=9$, $29 \%$ ), Enterococcus faecium ( $n=8,25 \%)$, and Pseudomonas aeruginosa $(n=5,16 \%)$.

\section{Risk factors for the development of infection}

Table 1 describes the main risk factors potentially associated with the development of nosocomial infection among COVID-19 patients. Most patients were treated with a course of ceftriaxone following admission $(n=120,86 \%)$ and/or azithromycin $(n=118,84 \%)$. Moreover, 105 patients $(75 \%)$ were given a course of another antibiotic treatment for a duration of at least 3 days. In addition, a high proportion of patients received therapy with corticosteroids (90\%) and/or tocilizumab (68\%) The development of infection was significantly associated with the APACHE II score at ICU admission, diabetes, and the use of corticosteroids (Table 1). In the multivariate analysis (including APACHE II, diabetes, and the use of steroids), only the APACHE II score was independently associated with the development of infection (odds ratio 1.09, 95\% confidence interval 1.02-1.17, $p=0.013$ ).

\section{Mortality and outcome}

Fifty-one (36\%) patients died in the ICU. All patients who were discharged alive from the ICU survived to hospital 
Table 3 Clinical characteristics and comparison between survivors and non-survivors

\begin{tabular}{|c|c|c|c|c|}
\hline & Patients $(N=140)$ & Non-survivors $(N=51)$ & Survivors $(N=89)$ & $p$ value \\
\hline Age-year (IQR) & $61(57-67)$ & $64(58-70)$ & $60(55-65)$ & 0.006 \\
\hline Sex-male & $108(77 \%)$ & $41(80 \%)$ & $67(75 \%)$ & 0.48 \\
\hline Body mass index (IQR) & $30.4(26-32)$ & $29.8(26-32)$ & $30.7(26-32)$ & 0.52 \\
\hline APACHE II (IQR) & $14(10-17)$ & $15.9(13-19)$ & $12.9(9-16)$ & 0.001 \\
\hline Hypertension & $60(42 \%)$ & $25(49 \%)$ & $39(43 \%)$ & 0.9 \\
\hline Chronic ischemic heart disease & $20(14 \%)$ & $9(17 \%)$ & $11(12 \%)$ & 0.39 \\
\hline Chronic obstructive pulmonary disease & $10(7 \%)$ & $5(10 \%)$ & $8(9 \%)$ & 0.9 \\
\hline Diabetes & $28(20 \%)$ & $9(17 \%)$ & $19(21 \%)$ & 0.59 \\
\hline ICU length of hospitalization - median (IQR), days & $14(8-17)$ & $18(11-21)$ & $13(7-16)$ & $<0.001$ \\
\hline Severe ARDS at ICU admission & $83(59 \%)$ & $40(78 \%)$ & $43(48 \%)$ & $<0.001$ \\
\hline Steroids & $127(90 \%)$ & $49(95 \%)$ & $78(87 \%)$ & 0.09 \\
\hline Tocilizumab & $96(68 \%)$ & $38(74 \%)$ & $58(65 \%)$ & 0.25 \\
\hline Nosocomial infection in the ICU & $57(40.7 \%)$ & $31(60 \%)$ & $26(29 \%)$ & $<0.001$ \\
\hline Septic shock & $38(27 \%)$ & $23(45 \%)$ & $15(17 \%)$ & $<0.001$ \\
\hline
\end{tabular}

Data are presented as number and \%, unless otherwise indicated. The most commonly used steroid dose regimen was methylprednisolone $1 \mathrm{mg} / \mathrm{kg} / \mathrm{die}$ (average duration 10 days). IQR interquartile range, $A R D S$ acute respiratory distress syndrome, $I C U$ intensive care unit

discharge. Table 3 presents the characteristics of patients who died compared with those who survived.

Mortality correlated significantly $(p<0.05)$ with a higher APACHE II score, the presence of severe ARDS at ICU admission, and the development of nosocomial infection at the ICU. Among the patients who had a nosocomial infection, 38 developed septic shock (27\%) during the course of their disease, which was significantly associated with a higher mortality $(p<0.001)$. In the multivariate analysis (including all the variables significantly associated with the mortality in the univariate analysis), the presence of severe ARDS at admission (OR $4.9,95 \%$ CI $2-12.2 ; p=0.001)$, the development of a nosocomial infection (OR 2.7, 95\% CI 1.2-5.9, $p=0.015$ ), and the APACHE II score (OR 1.1, CI 95\% 1.01-1.19; $p=0.017$ ) were all significantly associated with ICU mortality.

Infection was the main cause of death in 17 (33\%) of the 51 patients who died in the ICU, representing the second cause of death, after refractory respiratory failure (Table 3 ). ICU stay was significantly longer in patients with ICU infection $(p<0.001)$.

\section{Discussion}

We analyzed nosocomial infections acquired in the ICU in critically ill COVID-19 patients, during the main outbreak of the disease which hit Spain at the beginning of 2020. To our knowledge, this is the first series to specifically characterize the epidemiology, clinical presentation, and outcome of this cohort of ICU patients. We recorded a high incidence of nosocomial infections, which had a significant impact on hospital mortality, representing the main cause of death in $33 \%$ of the patients who died in the ICU.
The general characteristics of our population are similar to those described in other reports of COVID-19 in the ICU published so far [13-15]. Co-infections in COVID-19 have been described in previous studies (often within larger studies including all hospitalized COVID-19 patients), and reported incidence varies greatly, according to definition criteria, the heterogeneity of patients included, and the diagnostic methods used. We observed a higher incidence of nosocomial infections compared to those reported in some recent meta-analysis [5, 6, 16, 17]. However, when we compared our results with those of studies that only included COVID-19 patients hospitalized in the ICU, we observed more similar results with incidences ranging between 27 and $37 \%$ in several reports $[15,18-21]$. The occurrence of a nosocomial infection was a late complication, occurring after a median of more than 1 week of ICU stay.

We have observed a wide spectrum of nosocomial infections typically described in ICU patients such as VAP and tracheobronchitis, CRBSI, or UTI. However, it is noteworthy the high proportion of patients with primary BSI and CRBSI that represented $31 \%$ and $25 \%$ of infections, respectively. The high incidence of CRBSI could be explained by the strain put on the ICU and the whole hospital by the COVID-19 outbreak; in fact, during the peak of the COVID-19 outbreak, the ICU capacity of the hospital had to be increased by $350 \%$ in order to accommodate all patients in need of critical care. This entailed a high number of relocated healthcare personnel which often had to be employed in "non-conventional" temporary ICUs, such as operating theatres or post-anaesthesia care units. Moreover, infection control measures were strongly directed at avoiding the spread of airborne viral pathogens, and, during the peak of the pandemic, less attention could have been paid to ordinary infection control practices, and care bundles when handling IV lines 
or tracheal tubes [4]. The vast majority of our cohort received empiric antibiotic treatment with ceftriaxone at hospital admission, as was advocated by the early literature and guidelines [22-24]. However our data show that co-infection at presentation or during the first days of ICU stay was rare in our cohort. On the other hand, this very high exposure to antibiotic treatment could have facilitated subsequent infections during ICU stay, in particular by Gram positives such as enterococci [25].

Our data show that the only factor significantly associated with the development of nosocomial infection during ICU stay is the APACHE II score at admission. Immunomodulatory therapies have been used for the treatment of COVID-19, despite not being included in most treatment guidelines to this date for lack of convincing evidence [26]. In our sample, the most widely used immunomodulatory therapies were tocilizumab and glucocorticoids. Tocilizumab has been found to be associated with a significant increase in co-infections in cohorts of COVID-19 patients [27], but this finding was not confirmed in our study. The use of corticosteroids in COVID-19 is being largely investigated and is showing promising results. The cohort of patients receiving mechanical ventilation seems to be the one who benefits the most from the use of corticosteroids [28]. In our sample, corticoids were the only pharmacological treatment who was associated with the development of nosocomial infection (although not statistically significant in the multivariate analysis), and their use did not show any effect on mortality. The Recovery Trial which has shown the most promising data regarding the use of glucocorticoids in COVID-19 has not published results regarding the occurrence of nosocomial infection and their association with steroid treatment [28].

There are few data in the literature regarding the microbiology of bacterial/fungal co-infection in COVID-19. Some small studies have described the occurrence of infection sustained by MDR Gram-negative bacteria (Enterobacterales, A. baumannii, $P$. aeruginosa) $[5,17]$; others have found the commonest bacteria to be Mycoplasma pneumoniae, $P$. aeruginosa, $H$. influenzae, and Klebsiella spp. [6]. A recent single-centre study from Barcelona reported a similar microbiological pattern to the one we observed; however, this study included all hospitalized patients and did not differentiate between infections acquired in the ICU and in hospital wards [29].

It is noteworthy in our series the high incidence of BSI due to enterococci, with a remarkable predominance of $E$. faecium over $E$. faecalis, which may have been selected by the use of ceftriaxone as early antimicrobial treatment $[25,30]$. Moreover, Enterococcus spp. are not a major pathogen involved in nosocomial infections in Spanish ICUs [31]. The elevated prevalence of Enterococcus spp. has been highlighted by another study investigating BSI in ICU patients with COVID-19; it is noteworthy that a high percentage of that cohort of patients also received cephalosporins as empirical treatment [32]. The majority of our patients have also received courses of broad-spectrum antibiotics, in several cases without a confirmed diagnosis of nosocomial infection, and despite this large antimicrobial coverage, we recorded a high incidence of infections. Severe COVID-19 can easily mimic bacterial sepsis $[19,33]$, and this has certainly led many physicians to prescribe antibiotic treatment which then has proven not to be justified. This result shows that, during the peak of the outbreak, the principles of antimicrobial stewardship were implemented less strictly, a problem which has been highlighted in the literature [5, 6]. Our data do not support the use of antimicrobials in COVID-19 unless a bacterial/fungal superinfection is suspected. This is now in accordance with more recent literature and updated guidelines for the treatment of COVID-19 [5, 19]. Our data show a high incidence of VAP, with a strong predominance of Gram-negative bacteria; this is in accordance with previous reports of ICU patients with COVID-19 [20, 29].

We observed a high incidence of MDR bacteria (31\%). We recorded a high number of infections by fungi, and most importantly Aspergillus spp. A recent study [34] has shown an extremely high incidence of infection by Aspergillus spp. in COVID-19 patients hospitalized in the ICU. The authors diagnosed these infections by a systematic screening process and showed a significant association with patient clinical outcomes. Our results have not shown any association with clinical outcomes of Aspergillus spp. infection; however, our sample may be too small, and no systematic screening for this specific infection was in place during the study period.

The overall mortality in our study was $36 \%$, which is similar to the largest series of ICU patients published thus far [13-15]. APACHE II score and severity of ARDS were strongly associated with mortality, which is in line with what previous studies have shown in COVID-19 [14]. In our study, the development of a nosocomial infection during ICU stay was independently associated with mortality and it is noteworthy that septic shock was the main cause of the death in a third of the patients who died (17 of 51 cases). Few studies thus far have published data regarding the causes of death in the ICU; therefore, it is hard to compare these results with the literature.

\section{Study limitations}

We should acknowledge some limitations to this study. The sample size was small and the retrospective design reduces control over multiple confounders and data collection. We only included infections that were documented by culture and, therefore, some episodes may be missing. Finally, this study was limited to a single institution, with its own local epidemiology on antimicrobial resistance, which may limit the generalizability of the findings.

\section{Conclusions}

The role of COVID-19 in favouring bacterial superinfection is still a matter of debate. Our data show that patients who 
require ICU and advanced organ support tend to develop superinfection frequently and are at a significantly increased risk of death. Infection appears after more than 1 week of ICU stay and significantly prolongs the duration of ICU hospitalization. Bloodstream infections are a common occurrence and are frequently sustained by enterococci. In this context, measures to reduce bacterial infection are fundamental in order to provide appropriate critical care, and the implementation of antimicrobial stewardship programmes is of paramount importance.

Supplementary Information The online version contains supplementary material available at https://doi.org/10.1007/s10096-020-04142-w.

Authors' contributions Tommaso Bardi and Vicente Pintando the study, analyzed the data, and wrote the manuscript. Maria Gomez-Rojo, Rosa Escudero-Sanchez, Amal Azzam Lopez, Yolanda Diez-Remesal, Nilda Martinez Castro, Patricia Ruiz-Garbajosa, and David Pestaña collected the data and reviewed the manuscript.

Data availability The data of this study are available by contacting the corresponding author upon reasonable request.

\section{Compliance with ethical standards}

Conflict of interest The authors declare that they have no conflict of interest.

Ethics approval This study was approved by the hospital ethics committee.

\section{References}

1. Zhou F, Yu T, Du R et al (2020) Clinical course and risk factors for mortality of adult inpatients with COVID-19 in Wuhan, China: a retrospective cohort study. Lancet 395(10229):1054-1062. https:// doi.org/10.1016/S0140-6736(20)30566-3

2. Yang X, Yu Y, Xu J et al (2020) Clinical course and outcomes of critically ill patients with SARS-CoV-2 pneumonia in Wuhan, China: a single-centered, retrospective, observational study. Lancet Respir Med 8(5):475-481. https://doi.org/10.1016/S22132600(20)30079-5

3. Borobia AM, Carcas AJ, Arnalich F et al (2020) A cohort of patients with COVID-19 in a major teaching hospital in Europe. medRxiv. https://doi.org/10.1101/2020.04.29.20080853

4. Zhou P, Liu Z, Chen Y, Xiao Y, Huang X, Fan X-G Bacterial and fungal infections in COVID-19 patients: a matter of concern. Infect Control Hosp Epidemiol 1. https://doi.org/10.1017/ICE.2020.156

5. Rawson TM, Moore LSP, Zhu N et al (2020) Bacterial and fungal co-infection in individuals with coronavirus: a rapid review to support COVID-19 antimicrobial prescribing. Clin Infect Dis. https:// doi.org/10.1093/cid/ciaa530

6. Lansbury L, Lim B, Baskaran V, Lim WS Co-infections in people with COVID-19: a systematic review and meta-analysis. J Infect 0(0). https://doi.org/10.1016/J.JINF.2020.05.046

7. Horan TC, Andrus M, Dudeck MA (2008) CDC/NHSN surveillance definition of health care-associated infection and criteria for specific types of infections in the acute care setting. Am J Infect Control 36(5):309-332. https://doi.org/10.1016/j.ajic.2008.03.002

8. Chaves F, Garnacho-Montero J, del Pozo JL et al (2018) Diagnosis and treatment of catheter-related bloodstream infection: clinical guidelines of the Spanish Society of Infectious Diseases and Clinical Microbiology and (SEIMC) and the Spanish Society of Spanish Society of Intensive and Critical Care Medicine and Coronary Units (SEMICYUC). Med Int 42(1):5-36. https://doi. org/10.1016/j.medin.2017.09.012

9. Singer M, Deutschman CS, Seymour CW et al (2016) The third international consensus definitions for sepsis and septic shock (Sepsis-3). JAMA 315(8):801-810. https://doi.org/10.1001/jama. 2016.0287

10. Palomar M, Álvarez-Lerma F, Riera A, Díaz MT, Torres F, Agra Y, Larizgoitia I, Goeschel CA, Pronovost PJ, Bacteremia Zero Working Group (2013) Impact of a national multimodal intervention to prevent catheter-related bloodstream infection in the ICU: the Spanish experience. Crit Care Med 41(10):2364-2372. https:// doi.org/10.1097/CCM.0b013e3182923622

11. Álvarez-Lerma F, Palomar-Martínez M, Sánchez-García M, Martínez-Alonso M, Álvarez-Rodríguez J, Lorente L, AriasRivera S, García R, Gordo F, Añón JM, Jam-Gatell R, VázquezCalatayud M, Agra Y (2018) Prevention of ventilator-associated pneumonia: the multimodal approach of the Spanish ICU "Pneumonia Zero" Program. Crit Care Med 46(2):181-188. https://doi.org/10.1097/CCM.0000000000002736

12. Magiorakos A-P, Srinivasan A, Carey RB et al (2012) Multidrugresistant, extensively drug-resistant and pandrug-resistant bacteria: an international expert proposal for interim standard definitions for acquired resistance. Clin Microbiol Infect 18(3):268-281. https:// doi.org/10.1111/j.1469-0691.2011.03570.x

13. Grasselli G, Zangrillo A, Zanella A et al (2020) Baseline characteristics and outcomes of 1591 patients infected with SARS-CoV-2 admitted to ICUs of the Lombardy Region, Italy. JAMA 323(16): 1574. https://doi.org/10.1001/jama.2020.5394

14. Grasselli G, Greco M, Zanella A et al (2020) Risk factors associated with mortality among patients with COVID-19 in intensive care units in Lombardy, Italy. JAMA Intern Med. https://doi.org/10. 1001/jamainternmed.2020.3539

15. Ferrando C, Mellado-Artigas R, Gea A et al (2020) Características, evolución clínica y factores asociados a la mortalidad en UCI de los pacientes críticos infectados por SARS-CoV-2 en España: estudio prospectivo, de cohorte y multi-céntrico. Rev Esp Anestesiol Reanim. https://doi.org/10.1016/J.REDAR.2020.07.003

16. He Y, Li W, Wang Z, Chen H, Tian L, Liu D (2020) Nosocomial infection among patients with COVID-19: a retrospective data analysis of 918 cases from a single center in Wuhan, China. Infect Control Hosp Epidemiol 41(8):982-983. https://doi.org/10.1017/ ice. 2020.126

17. Langford BJ, So M, Raybardhan S et al (2020) Bacterial coinfection and secondary infection in patients with COVID-19: a living rapid review and meta-analysis. Clin Microbiol Infect 0(0). https://doi.org/10.1016/j.cmi.2020.07.016

18. Kreitmann L, Monard C, Dauwalder O, Simon M, Argaud L (2020) Early bacterial co-infection in ARDS related to COVID-19. Intensive Care Med 13:1-3. https://doi.org/10.1007/s00134-02006165-5

19. Youngs J, Wyncoll D, Hopkins P, Arnold A, Ball J, Bicanic T (2020) Improving antibiotic stewardship in COVID-19: bacterial co-infection is less common than with influenza. J Infect. https:// doi.org/10.1016/j.jinf.2020.06.056

20. Dudoignon E, Caméléna F, Deniau B et al (2020) Bacterial pneumonia in COVID-19 critically ill patients: a case series. Clin Infect Dis. https://doi.org/10.1093/cid/ciaa762

21. Rawson TM, Moore LSP, Zhu N et al (2020) Reply to Dudoignon et al. Clin Infect Dis. https://doi.org/10.1093/cid/ciaa767

22. Alhazzani W, Møller MH, Arabi YM et al (2020) Surviving Sepsis Campaign: guidelines on the management of critically ill adults with coronavirus disease 2019 (COVID-19). Intensive Care Med 46(5):854-887. https://doi.org/10.1007/s00134-020-06022-5 
23. Gautret P, Lagier J-C, Parola P et al (2020) Hydroxychloroquine and azithromycin as a treatment of COVID-19: results of an openlabel non-randomized clinical trial. Int J Antimicrob Agents 56(1): 105949. https://doi.org/10.1016/J.IJANTIMICAG.2020.105949

24. Phua J, Weng L, Ling L et al (2020) Intensive care management of coronavirus disease 2019 (COVID-19): challenges and recommendations. Lancet Respir Med 8(5):506-517. https://doi.org/10.1016/ S2213-2600(20)30161-2

25. Chakraborty R, Lam V, Kommineni S, Stromich J, Hayward M, Kristich CJ, Salzman NH (2018) Ceftriaxone administration disrupts intestinal homeostasis, mediating noninflammatory proliferation and dissemination of commensal enterococci. Infect Immun 86(12):e00674-e00618. https://doi.org/10.1128/IAI.00674-18

26. WHO Interim Guidance. Clinical management of COVID-19. 27 May. Published 2020. https://www.who.int/publications/i/item/ clinical-management-of-covid-19. Accessed 9 Aug 2020

27. Guaraldi G, Meschiari M, Cozzi-Lepri A et al (2020) Tocilizumab in patients with severe COVID-19: a retrospective cohort study. Lancet Rheumatol 2(8):e474-e484. https://doi.org/10.1016/ S2665-9913(20)30173-9

28. Group TRC Dexamethasone in hospitalized patients with Covid-19 — preliminary report. N Engl J Med:NEJMoa2021436. https://doi. org/10.1056/NEJMoa2021436

29. Garcia-Vidal C, Sanjuan G, Moreno-García E et al (2020) Incidence of co-infections and superinfections in hospitalised patients with COVID-19: a retrospective cohort study. Clin Microbiol Infect 0(0). https://doi.org/10.1016/j.cmi.2020.07.041

30. Papadimitriou-Olivgeris M, Drougka E, Fligou F et al (2014) Risk factors for enterococcal infection and colonization by vancomycinresistant enterococci in critically ill patients. Infection 42:10131022. https://doi.org/10.1007/s15010-014-0678-1

31. Estudio Nacional de Vigilancia de Infecciòn Nosocomial en Servicios de Medicina Intensiva (ENVIN). Nosocomial infections in Spanish ICU national registry. https://hws.vhebron.net/envinhelics/. Accessed 31 Aug 2020

32. Giacobbe DR, Battaglini D, Ball L et al (2020) Bloodstream infections in critically ill patients with COVID-19. Eur J Clin Invest. https://doi.org/10.1111/eci.13319

33. Li H, Liu L, Zhang D et al (2020) SARS-CoV-2 and viral sepsis: observations and hypotheses. Lancet (London, England) 395(10235):1517-1520. https://doi.org/10.1016/S0140-6736(20) 30920-X

34. Bartoletti M, Pascale R, Cricca M et al (2020) Epidemiology of invasive pulmonary aspergillosis among COVID-19 intubated patients: a prospective study. Clin Infect Dis. https://doi.org/10.1093/ $\mathrm{cid} / \mathrm{ciaa} 1065$

Publisher's note Springer Nature remains neutral with regard to jurisdictional claims in published maps and institutional affiliations. 